\title{
First two autochthonous dengue virus infections in metropolitan France, September 2010
}

G La Ruche (g.laruche@invs.sante.fr) ${ }^{1}$, Y Souarès ${ }^{1}$, A Armengaud ${ }^{2}$, F Peloux-Petiot ${ }^{3}$, P Delaunay4, P Desprès ${ }^{5}$, A Lenglet 6 ,

F Jourdain ${ }^{7}$, I Leparc-Goffart ${ }^{8}, \mathrm{~F}$ Charlet ${ }^{3}$, L Ollier $^{4}$, K Mantey $^{6}$, T Mollet $^{6}$, J P Fournier ${ }^{4}$, R Torrents ${ }^{2}$, K Leitmeyer ${ }^{6}$, P Hilairet ${ }^{4}$,

H Zeller ${ }^{6}$, W Van Bortel ${ }^{6}$, D Dejour-Salamanca ${ }^{1}$, M Grandadam ${ }^{5}$, M Gastellu-Etchegorry $^{1}$

1. French Institute for Public Health Surveillance (Institut de Veille Sanitaire, InVS), Saint-Maurice, France

2. Regional office of the French Institute for Public Health Surveillance (Cire Sud), Marseille, France

3. Regional Health Agency of Provence-Alpes-Côte d'Azur, Marseille and Nice, France

4. Entomology-Parasitology, Virology and Emergency Medicine and Internal Medicine Departments, University Hospital of Nice, Nice, France

5. Institut Pasteur, National Reference Centre for arboviruses, Paris, France

6. European Centre for Disease Prevention and Control (ECDC), Stockholm, Sweden

7. Directorate General for Health, Ministry of Health, Paris, France

8. Institut de recherche biomédicale des armées, National Reference Centre for arboviruses associated laboratory, Marseille, France

In September 2010, two cases of autochthonous dengue fever were diagnosed in metropolitan France for the first time. The cases occurring in Nice, southeast France, where Aedes albopictus is established, are evidence of dengue virus circulation in this area. This local transmission of dengue calls for further enhanced surveillance, active case finding and vector control measures to reduce the spread of the virus and the risk of an epidemic.

Dengue fever is the most important mosquito-borne viral disease in the world and is endemic in Africa, Asia, Caribbean and Latin America. According to the World Health Organization, there are annually more than 50 million cases and 22,000 deaths [1]. Dengue fever is caused by viruses of the Flaviviridae family and transmitted by mosquito vectors of the Aedes genus, mainly Ae. aegypti and Ae. albopictus [2].

In Europe, the last dengue epidemic was reported from 1927 to 1928 in Greece with high mortality and Ae. aegypti was implicated as the vector [3]. Since the 1970s, mainly through global trade of car tyres, Ae. albopictus has become increasingly established in European Union Member States, including France, Greece, Italy, the Netherlands (though only in greenhouses), Slovenia and Spain [4]. This mosquito species is also established in neighbouring countries such as Albania, Bosnia and Herzegovina, Croatia, Monaco, Montenegro, San Marino, Switzerland and Vatican City $[2,5]$. Imported cases of dengue fever in travellers returning from countries where dengue is endemic or where dengue epidemics are taking place have been frequently reported in European countries in recent years [6-10].
In metropolitan France, sporadic Ae. albopictus mosquitoes were first detected in Normandy in 1999 [11], but the mosquito is known to have been established since 2004 in south-east France [12]. Since 2006, and the widespread epidemic of chikungunya in Réunion which had posed an increased risk of importation of cases, enhanced surveillance is implemented each year from May to November in the departments where Ae. albopictus is established, as part of the national plan against the spread of chikungunya and dengue viruses in metropolitan France [13]. Enhanced surveillance, compared with routine surveillance, allows the reporting and confirmation of suspected cases to be accelerated. The laboratory network surveillance system, the most sensitive routine system in France, detected around 350-400 imported dengue cases per year between 2006 and 2009 in metropolitan France $[14,15]$. During the same four-year period, enhanced surveillance reported a total of 33 imported dengue cases (including 11 cases in 2009). Between 1 May and 17 September 2010 (i.e. the first 4.5 months of surveillance), 120 imported cases of dengue have been reported by the enhanced surveillance system [16], which represents an 11-fold increase when compared with the entire 2009 season. This increase in imported cases is mostly related to the ongoing epidemics in the French West Indies, Martinique and Guadeloupe, since the beginning of 2010. Here we report on the two first cases of autochthonous dengue virus infection ever diagnosed in metropolitan France and the public health measures subsequently implemented.

\section{Case 1}

The first case was detected through the routine enhanced surveillance system. The patient was a man 
in his 6os, resident in Nice, Alpes-Maritimes department, who developed fever, myalgia and asthenia on 23 August 2010. He was hospitalised on 27 August 2010, but his clinical condition remained stable. A temporary thrombocytopenia with a minimal platelet count of $48,000 / \mu \mathrm{l}$ (norm: 150,000-400,000) on day five of the illness resolved without complications and he recovered within a few days after disease onset.

\section{Laboratory findings}

A panel of sera obtained during the acute and recovery phases on days five, seven, 11 and 25 of the illness was investigated by serological tests (in-house MAC-ELISA and direct IgG ELISA) and real-time RT-PCR. Moreover, a serum sample collected during a previous medical examination in May 2010 was tested retrospectively. Presence of IgM and IgG against dengue virus antigens was documented in all samples except for the serum sampled in May 2010. Antibody titration revealed sharp increases in IgM titres from $1: 800$ to $1: 12,800$ and in IgG titres from $1: 32,000$ to $>1: 128$,000 over the 25 days follow-up. Anti-dengue virus IgA (Assure Dengue IgA rapid test, MP Biomedicals) were also detected on days five and seven. The dengue NS1 antigenic test (Dengue NS1 strip, Biorad) was positive on days five and seven but negative on day 11, demonstrating the active replication of a dengue virus during the symptomatic period. RT-PCR for dengue virus was positive on day five and negative thereafter. Molecular typing identified a dengue virus serotype 1.

It is of interest to note that high levels of specific antidengue IgG were detected during the acute phase of disease. Our hypothesis is that these IgG might result from activation of memory $B$ cells (original antigenic sin) related to an ancient primary infection with a heterologous serotype of dengue virus. Seroneutralisation tests will be informative on the immunological status of the patient regarding a possible previous infection with a dengue virus of another serotype. Virus isolation and sequencing are also ongoing. No serum crossreactions were observed with tick-borne encephalitis and West Nile viruses and no markers of chikungunya virus infection were found (absence of IgM and IgG antibodies, negative RT-PCR). The patient had been vaccinated against yellow fever 28 years ago.

Friends from the French West Indies had stayed with him since April 2010. He had no recent history of international travel or blood transfusion. Consequently, the patient was considered a confirmed autochthonous case of dengue virus infection.

\section{Control measures}

This classification prompted an immediate reaction of public health authorities to reduce the risk of further spread of the virus. Various measures were undertaken by health authorities as laid out in the national plan against the spread of dengue in France (level 2 of the plan) [13]: (i) 200 metres perifocal vector control activities centred on the case's residence, including spraying for adult mosquitoes and destruction of breeding sites; (ii) active case finding in the neighbourhood of the case's residence and in other areas visited by the case; (iii) providing information about dengue virus to health professionals, including incitation for screening suspected dengue cases and information to the public. The active case finding conducted by physicians and laboratories will be continued on a weekly basis up to 45 days after the onset of symptoms of the last autochthonous case.

The routine laboratory network surveillance system noticed that six recently imported confirmed dengue cases, including four with a RT-PCR positive for dengue, had been detected in Nice between 24 July and 23 August 2010. One of them had returned from Martinique and lives about 200 metres from the autochthonous case. This imported case was reported too late to implement vector control measures which routinely follow imported viraemic dengue cases in those departments where the vector is present, and could therefore be a potential source of infection of local Ae. albopictus. As of 24 September 2010, the active case finding has detected nine new suspected autochthonous cases of dengue fever in the neighbourhood of the index case. In four of them, no markers of dengue virus infection were found (absence of IgM and IgG antibodies, negative RT-P(R), results from epidemiological and laboratory investigations for further four patients are still pending. One case was confirmed to be infected by dengue virus; the latter patient is the second autochthonous dengue fever case ever diagnosed in metropolitan France.

\section{Case 2}

This second case is an 18 year-old man who had no recent history of international travel. He lives approximately 70 metres from the first autochthonous case. He developed fever, myalgia, headache and asthenia on 11 September 2010. He was hospitalised briefly because of fever of unknown origin and thrombocytopenia with a mild clinical disease. The thrombocytopenia (platelet count $53,000 / \mu \mathrm{L}$ on day seven of the illness) was temporary and moderate, and he has recovered fully.

\section{Laboratory investigations}

Laboratory tests conducted on an early serum sample on day three of illness indicate negative serology for IgG and IgM antibodies but strongly positive RT-PCR for dengue virus. Molecular typing identified a dengue virus serotype 1 . The strain appears to be quite similar to those which currently circulate in Martinique; more detailed analyses are ongoing.

\section{Discussion}

The identification of two autochthonous cases of dengue fever which are clustered in space and time is strongly suggestive that a local transmission of dengue virus is ongoing. Therefore level 3 of the national plan against the spread of dengue virus has been activated [13]. It entails additional measures to those taken 
at level 2: (i) active case finding of autochthonous cases in hospital emergency wards, at present in Nice and surrounding towns, (ii) implementation of vector control measures in hospitals, together with protection of potential viraemic patients against mosquito bites using electric light traps, electric diffusers for insecticides, and repellents, and vector control measures around the port and the international airport of Nice including enhanced entomological surveillance, and (iii) toxicovigilance related to the wide use of insecticides.

Based on the currently available information, these are the first confirmed cases of autochthonous transmission of dengue fever in metropolitan France and Europe, since the epidemic in Greece in the late $1920 \mathrm{~s}$ and apart from one nosocomial case of dengue infection reported from Germany in 2004 [17]. The event is not entirely unexpected, as reflected in a specific preparedness plan and taking into account the increase in imported cases from the French West Indies and other endemo-epidemic areas. It is known that France, as well as other countries in Europe, has competent vectors for transmitting this flavivirus. The chikungunya outbreak in Italy that occurred in 2007 , with over 300 cases reported, has shown that non-endemic arboviruses can be efficiently transmitted in continental Europe [18].

Whether the transmission of dengue virus in France followed a bite from an infectious mosquito imported to the area via airplanes or boats, or one already present in the area after biting a viraemic person residing or visiting Nice, remains to be determined. However, with the second confirmed case, the latter scenario is the most likely one. Therefore, taking into consideration the longest possible incubation period for dengue fever, 15 days, it can be considered that the conditions for successful transmission of dengue virus to humans existed in Nice during August 2010. To date, only two autochthonous cases of dengue fever have been detected in Nice, but the identification of new dengue cases in the near future cannot be excluded. The enhanced surveillance and strict vector control measures are expected to limit the risk for further spread as much as possible.

At this stage, the risk for further spread to humans in Europe, as well as the possibility of the establishment of dengue virus transmission in Nice or in neighbouring areas in France, may appear limited but needs to be closely monitored. Recent evidence demonstrates that compared with Ae. aegypti, which has been implicated in the majority of large dengue outbreaks worldwide, Ae. albopictus is a less efficient vector of this virus [2]. Nevertheless, it was involved in outbreaks in Japan from 1942 to 1945 [19], the Seychelles in 1977 [20], Hawaii from 2001 to 2002 [21] and Réunion island in 2004 [22]. Vertical transmission of dengue virus from mosquitoes to their offspring does not seem very efficient, and therefore overwintering of the virus in continental European Ae. albopictus populations is unlikely [2] but cannot be excluded [23,24]. The public health consequences of the presence of Ae. albopictus, in this context, appear to be more important for the transmission of chikungunya for example, for which experimentally better competence has been demonstrated, although the competence of local Ae. albopictus for dengue virus is far from being negligible [25]. It should also be noted, that the currently affected area of France as well as other countries in Europe is faced with a high number of imported dengue cases every year. However, despite this and established mosquito populations being potentially able to transmit arboviral diseases, local transmission of the dengue virus with Ae. albopictus as the vector in mainland Europe has never been observed before this reported emergence in the south-east of metropolitan France. The high vector density in Nice and the increase in the number of imported cases in this area in 2010, mainly due to intense epidemics in the French West Indies, are two major factors to explain this emergence and highlight the need to maintain an appropriate active surveillance.

In terms of blood safety, reported dengue infection following blood transfusion in dengue endemic areas is rare [26-28] but is also difficult to detect as a large proportion of the population would already have antibodies against the virus. However, as dengue infection is mild or asymptomatic in $40-80 \%$ of infected persons, depending on the area and the epidemiological context [29-31], it does pose a risk to blood safety. The two identified cases in Nice are suggestive that other infected persons may have lived in the city during the same period of exposure, without showing any symptoms. Asymptomatic carriers of dengue virus could pose a potential risk to blood safety if they donate blood while being viraemic. It is possible however, that the duration of viraemia in mild or asymptomatic cases is shorter and the virus titre is lower than in symptomatic persons, but this hypothesis is far from proven. Moreover, the limited extend of current virus dissemination, as shown by the actual clustering of confirmed autochthonous cases, does not indicate that such asymptomatic infections could have been spread around the whole city of Nice. At present, it is difficult to quantify this risk, and only a retrospective survey of blood supplies from Nice between July and September 2010 would allow to estimate it better. In France, authorities in charge of blood routinely exclude all febrile donors from donation. No additional exclusion measures have been implemented after the two neighbouring cases as the risk for dengue transmission has been considered very low.

Further investigations to identify the likely source of exposure of the two cases, as well as extensive comparison of the dengue virus genotypes between the locally identified viruses and the strains currently circulating in the French West Indies, will hopefully allow a better understanding of this event. The reactive surveillance in addition to the routine enhanced surveillance 
is likely to identify new symptomatic cases in the area, determining also the potential geographic extension of the risk. Finally, better understanding is needed on how the vector abundance, activity and competence of Ae. albopictus for dengue transmission influence the risk for further transmission in the region [25,32].

\section{Conclusion}

The current clustering of cases of locally transmitted dengue fever in Nice is a significant public health event, but is not unexpected and more cases can be predicted. Such transmission was anticipated by the development of a national plan. Although this plan should be adjusted in the light of this experience, this event shows the advantage of such preparedness in order to implement rapid and proportionate measures of surveillance and control. Previous events, including a mosquito-borne arbovirus outbreak in Italy, the occurrence of vector-borne diseases around airports and other ports of entry and a previous risk assessment on dengue virus introduction in European Union countries [4] indicate that autochthonous transmission in continental Europe is possible, as confirmed by the present event. However, according to the available epidemiological information, the risk for establishment of dengue transmission in south-eastern France or further spread in Europe currently appears limited. Further data available in the near future will allow us to re-assess this likelihood.

\section{Acknowledgements}

We thank the biomedical laboratories involved in the enhanced surveillance: Cerba (Saint-Ouen l'Aumone), Biomnis (Lyon), La Timone (Marseille) and Avicenne hospital (Bobigny), part of the national laboratory surveillance system, and the private laboratories in south-east France.

\section{References}

1. World Health Organization (WHO). Impact of dengue. Geneva: WHO. [Accessed 27 Sep 2010]. Available from: http://www. who.int/csr/disease/dengue/impact/en/index.html

2. Lambrechts L, Scott TW, Gubler DJ. Consequences of the expanding global distribution of Aedes albopictus for dengue virus transmission. PLoS Negl Trop Dis. 2010;4(5):e646.

3. Halstead SB, Papaevangelou G. Transmission of dengue 1 and 2 viruses in Greece in 1928. Am J Trop Med Hyg. 1980;29(4):635-7.

4. Seyler T, Grandesso F, Le Strat Y, Tarantola A, Depoortere E. Assessing the risk of importing dengue and chikungunya viruses to the European Union. Epidemics 2009;1(3):175-184.

5. European Centre for Disease Prevention and Control (ECDC). Development of Aedes albopictus risk maps. Stockholm: ECDC; 2009. Available from: http://www.ecdc.europa.eu/en/ publications/Publications/0905_TER_Development_of_Aedes_ Albopictus_Risk_Maps.pdf

6. Nisii C, Carletti F, Castilletti C, Bordi L, Meschi S, Selleri M, et al. A case of dengue type 3 virus infection imported from Africa to Italy, October 2009. Euro Surveill. 2010;15(7). pii: 19487. Available from: http://www.eurosurveillance.org/ViewArticle. aspx?Articleld $=19487$

7. Jelinek T. Trends in the epidemiology of dengue fever and their relevance for importation to Europe. Euro Surveill. 2009;14(25). pii: 19250. Available from: http://www.eurosurveillance.org/ ViewArticle.aspx?Articleld $=19250$

8. Pinazo MJ, Muñoz J, Betica L, Maretic T, Zekan S, Avsic-Zupanc T, et al. Imported dengue hemorrhagic fever, Europe. Emerg Infect Dis. 2008;14(8):1329-30.
9. Wichmann O, Gascon J, Schunk M, Puente S, Siikamaki H, Gjørup I, et al. Severe dengue virus infection in travelers: risk factors and laboratory indicators. I Infect Dis. 2007;195(8):1089-96.

10. Gautret P, Simon F, Hervius Askling H, Bouchaud O, LeparcGoffart I, Ninove L, et al. Dengue type 3 virus infections in European travellers returning from the Comoros and Zanzibar, February-April 2010. Euro Surveill. 2010;15(15). pii: 19541. Available from: http://www.eurosurveillance.org/ViewArticle. aspx?Articleld $=19541$

11. Schaffner F, Karch S. [First report of Aedes albopictus (Skuse, 1984) in metropolitan France]. C R Acad Sci III. 2000;323(4):373-5. French.

12. Delaunay P, Mathieu B, Marty P, Fauran P, Schaffner F. [Chronology of the development of Aedes albopictus in the Alpes-Maritimes Department of France, from 2002 to 2005]. Med Trop (Mars). 2007;67(3):310-1.

13. Ministère de la santé et des sports. Circulaire Nnº DGS/ $\mathrm{Rl} 1 / 2010 / 163$ du 17 mai 2010 relative aux modalités de mise en œuvre du plan anti dissémination du chikungunya et de la dengue en métropole. French. Available from: http://www. circulaires.gouv.fr/pdf/2010/05/cir_31164.pdf

14. La Ruche G, Dejour Salamanca D, Debruyne M, Leparc-Goffart I, Ledrans M, Grandadam M, et al. [Laboratory surveillance of dengue and chikungunya cases imported in metropolitan France 2008-2009]. Bull Epidemiol Hebdo. 2010;(3132):325-9. French. Available from: http://www.invs.sante.fr/ beh/2010/31_32/index.htm

15. Dejour Salamanca D, La Ruche G, Tarantola A, Souares Y, Armengaud A, Peloux-Petiot F, et al. [Reported dengue cases in metropolitan France 2006-2008: need for the improvement of reporting]. Bull Epidemiol Hebdo. 2010;(11):101-4. French. Available from: http://www.invs.sante.fr/beh/2010/11/index. htm

16. Cellule de l'Institut de veille sanitaire en région (Cire) Sud. [Surveillance of Chikungunya, Dengue, West-Nile, Toscana

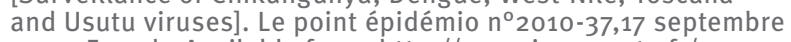
2010. French. Available from: http://www.invs.sante.fr/ regions/sud/pe_paca_corse_170910.pdf

17. Wagner D, de With K, Huzly D, Hufert F, Weidmann M, Breisinger S, et al. Nosocomial acquisition of dengue. Emerg Infect Dis. 2004;10(10):1872-3.

18. Angelini R, Finarelli AC, Angelini P, Po C, Petropulacos K, Silvi G, et al. Chikungunya in north-eastern Italy: a summing up of the outbreak. Euro Surveill. 2007;12(47). pii: 3313. Available from: http://www.eurosurveillance.org/ViewArticle. aspx?Articleld $=3313$

19. Hotta S. Dengue epidemics in Japan, 1942-1945. J Trop Med Hyg. 1953;56(4):83.

20. Effler PV, Pang L, Kitsutani P, Vorndam V, Nakata M, Ayers T, et al. Dengue fever, Hawaii, 2001-2002. Emerg Infect Dis. 2005;11(5):742-9.

21. Zeller HG. [Dengue, arbovirus and migrations in the Indian Ocean]. Bull Soc Pathol Exot. 1998;91(1):56-60. French.

22. Delatte H, Paupy C, Dehecq JS, Thiria J, Failloux AB, Fontenille D. [Aedes albopictus, vector of chikungunya and dengue viruses in Reunion Island: biology and control]. Parasite 2008;15(1):3-13. French.

23. Lee HL, Rohani A. Transovarial transmission of dengue virus in Aedes aegypti and Aedes albopictus in relation to dengue outbreak in an urban area in Malaysia. Dengue Bulletin 2005;29:106-11. Available from: http://www.searo.who.int/ LinkFiles/Dengue_Bulletins_Volumes_29_(2005)_CHAPTER13. pdf

24. Thenmozhi V, Hiriyan JG, Tewari SC, Philip Samuel P, Paramasivan R, Rajendran R, et al. Natural vertical transmission of dengue virus in Aedes albopictus (Diptera: Culicidae) in Kerala, a southern Indian state. Jpn J Infect Dis 2007;60(5):245-9.

25. Moutailler S, Barré H, Vazeille M, Failloux AB. Recently introduced Aedes albopictus in Corsica is competent to Chikungunya virus and in a lesser extent to dengue virus. Trop Med Int Health. 2009;14(9):1105-9.

26. Tambyah PA, Koay ES, Poon ML, Lin RV, Ong BK; TransfusionTransmitted Dengue Infection Study Group. Dengue hemorrhagic fever transmitted by blood transfusion. N Engl J Med. 2008;359(14):1526-7.

27. Linnen IM, Vinelli E, Sabino EC, Tobler LH, Hyland C, Lee TH, et al. Dengue viremia in blood donors from Honduras, Brazil, and Australia. Transfusion. 2008;48(7):1355-62.

28. Chuang VW, Wong TY, Leung YH, Ma ES, Law YL, Tsang OT, et al. Review of dengue fever cases in Hong Kong during 1998 to 2005. Hong Kong Med J. 2008;14(3):170-7. 
29. Cobelens FG, Groen J, Osterhaus AD, Leentvaar-Kuipers A, Wertheim-van Dillen PM, Kager PA. Incidence and risk factors of probable dengue virus infection among Dutch travellers to Asia. Trop Med Int Health. 2002;7(4):331-8.

30. Burke DS, Nisalak A, Johnson DE, Scott RM. A prospective study of dengue infections in Bangkok. Am J Trop Med Hyg. 1988;38(1):172-80.

31. Porter KR, Beckett CG, Kosasih H, Tan RI, Alisjahbana B, Rudiman PI, et al. Epidemiology of dengue and dengue hemorrhagic fever in a cohort of adults living in Bandung, West Java, Indonesia. Am J Trop Med Hyg. 2005;72(1):60-6.

32. Talbalaghi A, Moutailler S, Vazeille M, Failloux AB. Are Aedes albopictus or other mosquito species from northern Italy competent to sustain new arboviral outbreaks? Med Vet Entomol. 2010;24(1):83-7. 